\title{
Development and Validation of RP-HPLC-DAD Method to Quantify Hydroxytyrosol Content in a Semi-Solid Pharmaceutical Formulation
}

\section{Antonella Smeriglio1, Corrado Giovinazzo² and Domenico Trombetta ${ }^{1 *}$}

${ }^{1}$ Dipartimento di Scienze del Farmaco e dei Prodotti per la Salute, Università di Messina, Villaggio S.S. Annunziata 98168, Messina, Italy ${ }^{2} P \& P$ Farma, Torino, Italy

\begin{abstract}
Many substances of natural origin are contained in pharmaceutical and cosmetic formulations, but lately extra virgin olive oil (EVOO) or EVOO-derived compounds like tyrosol, hydroxytyrosol or oleuropein, thanks to their renowned and long-established outstanding therapeutic and health giving virtues, have made them some of the most interesting products in this field. Nowadays many hydroxytyrosol-based topical formulations are commercialized and over time several analytical methods have been developed. However, new cosmetic formulations containing an olive extract tritated in hydroxytyrosol conveyed in EVOO have recently appeared on the market creating the need to develop and validate a new method that allows the active compound to be extracted and analyzed precluding any interference due to the presence of other compounds naturally present in the EVOO as well as from the co-formulant agents employed, and this has been the goal of our study.

Analytical determination was performed by RP-HPLC coupled with DAD detection. Various chromatographic parameters, namely column stationary phase, mobile phase, $\mathrm{pH}$ and solvent composition, oven temperature and different clean-up variables were studied. The best chromatographic separation was obtained under the following conditions: a reverse phase $\mathrm{C} 18$ column maintained at $25^{\circ} \mathrm{C}$ with a gradient elution program using acetic acid $0.2 \%$ and methanol as mobile phase pumped at $1.5 \mathrm{~mL} \mathrm{~min}^{-1}$. Detection wavelength was set at $280 \mathrm{~nm}$ and the total run time required was $15 \mathrm{~min}$. The high degree of accuracy (98.8\%-100.1\%) and precision (1.44\%-1.68\%) achieved using the evaluated method along with the low limits of detection and quantification ( $2.49 \mathrm{ppm}$ and $3.97 \mathrm{ppm}$ respectively) and the broad linear range observed allowed the target analyte to be satisfactorily determined in new semi-solid formulation containing hydroxytyrosol conveyed in EVOO while avoiding any matrix effect.
\end{abstract}

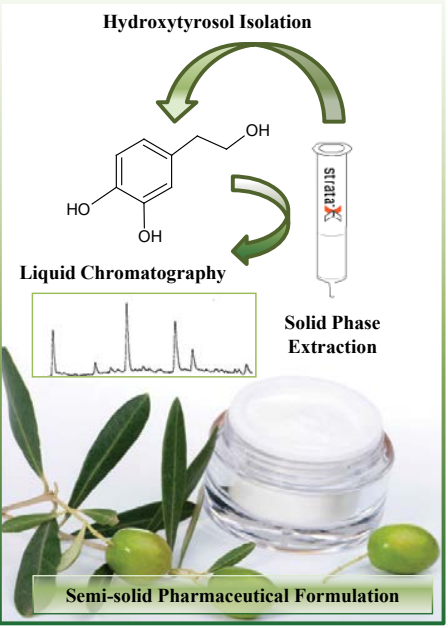

Keywords: Hydroxytyrosol; Extra virgin olive oil; Semi-solid formulations; Solid phase extraction; Liquid chromatography; Method validation

\section{Introduction}

There is now increasing scientific evidence to support the hypothesis that some foods and food components have beneficial physiological and psychological effects over and above the provision of basic nutrients. Research focus has shifted increasingly towards the identification of biologically active components in foods that have the potential to optimize physical and mental well-being and may also reduce the risk of disease. The aim is to increase life expectancy and to improve the quality of life while reducing healthcare costs $[1,2]$.

Extra Virgin Olive Oil (EVOO) represents a rich source of nutrient and non-nutrient compounds. From the non-nutrient fraction, representing about $1-2 \%$ of EVOO composition, polyphenols have always aroused interest not only because they are partly responsible for the stability of EVOO against autoxidation and its organoleptic characteristics, but also because several EVOO polyphenols seem

*Corresponding author: Domenico Trombetta, Dipartimento di Scienze del Farmaco e dei Prodotti per la Salute, Università di Messina, Villaggio S.S. Annunziata 98168 Messina, Italy, Tel: +39 0906766458; E-mail: dtrombetta@unime.it

Received September 18, 2015; Accepted October 05, 2015; Published October 07, 2015

Citation: Smeriglio A, Giovinazzo C, Trombetta D (2015) Development and Validation of RP-HPLC-DAD Method to Quantify Hydroxytyrosol Content in a Semi-Solid Pharmaceutical Formulation. Med chem 5: 442-446. doi:10.4172/2161 0444.1000298

Copyright: (c 2015 Smeriglio A, et al. This is an open-access article distributed under the terms of the Creative Commons Attribution License, which permits unrestricted use, distribution, and reproduction in any medium, provided the original author and source are credited. 
to explain some important effects on human health by interacting selectively with one or more physiological functions of the organism (biomodulation) thereby improving a person's state of health and/or reducing risk of disease [3-6].

Unlike other vegetable oils, EVOO is obtained from fruits and not from seeds; the seeds are dormant organisms with low metabolic activity while the fruit has high metabolic activity, which gives to the derived product greater complexity. Another difference is that it is not produced by solvent extraction, but rather by a cold-press mechanical process that preserves both its chemical nature and the natural antioxidants produced by the plant in response to environmental stress.

EVOO is one of the main ingredients of the Mediterranean diet and the European and Food Safety Authority (EFSA) has officially recognized its health properties by publishing a scientific opinion on the substantiation of health claims related to polyphenols [7]. EVOO contains a large number of phenolic compounds, including phenolic acids, phenolic alcohols such as tyrosol and hydroxytyrosol, flavonoids, and secoiridoids that may contribute to its overall health benefits together with monounsaturated fatty acids, squalene and a-tocopherol [8-11]. Polyphenols, in particular, exhibit potent free radical-scavenging properties and a range of biological activities e.g., antimicrobial, anti-carcinogenic and anti-inflammatory responses [12]. According to the EFSA statements, the oleuropein complex contributes significantly to the polyphenolic profile of EVOO and although this decreases over time, it remains fairly high due to the formation of degradation products such as oleuropein and tyrosol from more complex molecules.

There is currently a great deal of interest in the health benefits of hydroxytyrosol and its metabolites (Figure 1) [13]. It would seem that this small molecule, in its natural state or as a hydrolysis product of several polyphenols, is easily absorbed in the intestine via a passive diffusion mechanism, bidirectionally and in a dose dependent manner [14].

Hydroxytyrosol influences the melanin pigments in human cells, lightening the skin and making dark patches disappear, showing interesting anti-aging effects on skin and can also prevent wrinkle formation [15]. Recently, Salucci et al. [16,17] suggested that Tyrosol and Hydroxytyrosol play an important role in protecting cells from apoptotic cell death, thus encouraging the use of these phytochemicals as biological ingredients in topical preparations as a possible tool to prevent skin damage. Most skin lighteners currently used are of botanical or natural origin. Hydroxytyrosol seems to work by enhancing the body's own antioxidant ability, increasing the intracellular glutathione levels which shifts melanin production towards the more soluble and lighter pheomelanin and at the same time lightens skin color significantly by reducing overall melanin production [18].

In nature, hydroxytyrosol is found in olives and olive leaves and its extraction is complex and costly. Therefore, normally native olive oil has often been used in cosmetic formulations.

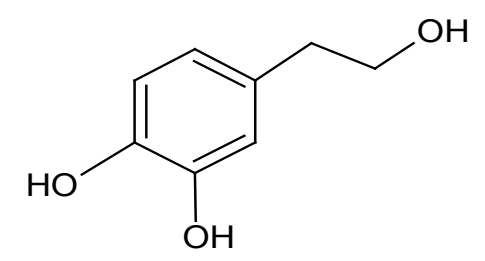

Figure 1: Chemical structure of hydroxytyrosol.
Hydroxytyrosol has often been incorporated in pharmaceutical and cosmetics formulations due to its higher antioxidant potential compared to other polyphenols with similar structures or other antioxidant molecules such as Vitamin E or C [19] in the form of olive leaf extract or recently as olive extract titrated in hydroxytyrosol and conveyed in EVOO. Hydroxytyrosol conveyed in EVOO would appear to be much more bioavailable than other formulations; probably also as a result of the synergic effect explicated by other naturally present polyphenols [20].

The peculiar characteristics of EVOO i.e., oxidative stability and sensory health-giving properties derive from its remarkable and wellbalanced chemical composition [3]. This is beneficial not only to human skin but also conserves its shelf life when used in emulsions and cream bases.

In light of the above, the aim of our research was to develop a validated, selective and reproducible extraction procedure and RPHPLC-DAD method to evaluate hydroxytyrosol content in a semisolid pharmaceutical formulation.

\section{Materials and Methods}

\section{Chemicals}

Hydroxytyrosol (2-(3,4-di-hydroxyphenyl)ethanol) $\geq 98 \%$ was purchased from Extrasynthese (Genay, France) and used as reference compound. Glacial acetic acid, methanol, acetonitrile and ethanol were HPLC grade and purchased from Merck (Darmstadt, Germany). Sulfuric acid was purchased from Sigma-Aldrich (Milan, Italy). Water used in all experiments was passed through a Milli-Q (Millipore, Milan, Italy) water purification system $(18 \mathrm{~m} \Omega)$.

\section{Apparatus}

An Agilent high performance liquid chromatography system (1100 series) equipped with an LC binary pump, an autosampler, a degasser and a thermostated column oven coupled with a UV-Vis photodiodearray detector (DAD) was used. The column used was an Ascentis C18 $(150 \times 4.6 \mathrm{~mm}, 5 \mu \mathrm{m})$ (Supelco, Milan, Italy).

An ultrasonic bath $(117 \mathrm{~V}, 60 \mathrm{~Hz})$ from VWR (USA) was used to facilitate the extraction procedure of the active compound.

An Agilent Vac Elut 20 Manifold was used for solid phase extraction (SPE) and a $30 \mathrm{mg}$ Strata-X cartridge (Phenomenenx) was employed. A Basic $20 \mathrm{pH}$ meter from Crison (Alella, Spain) was used for $\mathrm{pH}$ adjustments.

\section{Extraction procedure}

A semi-solid pharmaceutical formulation containing hydroxytyrosol as active compound conveyed in EVOO was subjected to the extraction procedure. Briefly, $1.0 \mathrm{~g}$ of sample was weighed in a 10 $\mathrm{mL}$ volumetric flask to which was added a mixture consisting of acetic acid $0.2 \%$ and methanol $75 / 25(\mathrm{v} / \mathrm{v})(\mathrm{pH} 3.30)$. This solution was placed in an ultrasonic water bath for five minutes to facilitate the dissolution of sample and relative extraction process.

A $500 \mu \mathrm{l}$ aliquot of the above solution was added to $1.5 \mathrm{ml}$ sulfuric acid $50 \mathrm{mM}$. Solid phase extraction (SPE) to purify the sample solution was carried out on a Strata-X $33 \mu \mathrm{m}$ polymeric reversed phase, 30 $\mathrm{mg} / \mathrm{mL}$ (Phenomenex, Germany). The cartridge was placed in a vacuum system device fitted with a small vacuum pump. Pressure was monitored during the critical cartridge conditioning, equilibration, and sample loading steps, thereby preventing fluctuations in separation efficiency due to variations in flow rate. The cartridge was conditioned at a flow rate of $2-3 \mathrm{~mL} / \mathrm{min}$, and the analyte was loaded, washed, and 
eluted at $1.0-1.5 \mathrm{~mL} / \mathrm{min}$. Prior to use, the cartridge was conditioned with $1 \mathrm{~mL}$ of methanol followed by equilibration with $1 \mathrm{~mL}$ of $\mathrm{H}_{2} \mathrm{O}$.

The sample solution, prepared as described above, was loaded slowly into the cartridge. The SPE cartridge was washed with $0.5 \mathrm{~mL}$ of water followed by $1 \mathrm{~mL}$ of $5 \%(\mathrm{v} / \mathrm{v})$ methanol in water and then eluted under vacuum with $1.5 \mathrm{~mL}$ of methanol. The eluted fraction was filtered through a $0.22 \mu \mathrm{m}$ nylon membrane filter and placed in a 1.5 $\mathrm{mL}$ injection vial.

A $500 \mu \mathrm{g} \mathrm{mL}^{-1}$ standard stock solution containing hydroxytyrosol was prepared using a mixture of acetic acid $0.2 \%$ and methanol $75 / 25$ (v/v) ( $\mathrm{pH} 3.30)$. From this solution, freshly working standard solutions from 3 to $100 \mu \mathrm{g} \mathrm{mL}^{-1}$ were prepared in the same solvent.

\section{Chromatographic analysis}

The analysis was performed following the method reported by Miralles et al. [21] with some modifications. $20 \mu \mathrm{L}$ of the reference compound or sample solution was injected into the column. The column oven was set at $25^{\circ} \mathrm{C}$. The mobile phase included a solvent $\mathrm{A}$, consisting of acetic acid $0.2 \%$ and solvent B methanol. Elution was performed at a flow rate of $1.5 \mathrm{~mL} \mathrm{~min}{ }^{-1}$. Separation was performed using the following gradient elution program: $0 \mathrm{~min}, 95 \% \mathrm{~A}$ and $5 \%$ B; $10.0 \mathrm{~min}, 75 \% \mathrm{~A}$ and $35 \% \mathrm{~B} ; 13.0 \mathrm{~min}, 95 \% \mathrm{~A}$ and $5 \% \mathrm{~B} ; 15.0 \mathrm{~min}$, 95.0\% A and 5.0\% B. Hydroxytyrosol was recognized and quantified at $280 \mathrm{~nm}$ based on the supplier's standard, comparing retention time and relative UV-VIS spectra (range $200-400 \mathrm{~nm}$ ).

\section{Method validation}

For validation of analytical method, the guidelines of the International Conference on the Harmonization of Technical Requirements for the Registration of Pharmaceuticals for Human Use [22] and the United States Pharmacopeial Convention [23] recommend the accomplishment of linearity and range, accuracy and precision, LOD and LOQ and selectivity.

Linearity and range: Linearity is the ability of a method to elicit test results that are directly proportional to the analyte concentration within a given range. Five concentration levels used along with certain minimum specified ranges are required. The acceptance criterion for linearity is that the correlation coefficient $\left(\mathrm{r}^{2}\right)$ should not be less than 0.990 for the least squares method of the analysis of the line [24]. To evaluate the linearity of the method, several concentrations of hydroxytyrosol reference compound were analyzed by HPLC$\mathrm{DAD}$, and the responses were recorded. Plotting the peak areas of the hydroxytyrosol versus concentration (in $\mathrm{ppm}$ ), the method was found (Figure 2) to be linear in the range of $3-100 \mathrm{ppm}$ with $\mathrm{r}^{2}>0.999$.

Accuracy (percentage recovery) and precision: Accuracy of the method was measured as the percentage of analyte recovered after spiking sample in a blank solution. To determine accuracy, a minimum of nine determinations of three different concentration levels covering the specified range (three concentrations, three replicates for each) were collected. The relative standard deviation (RSD) of the replicates provides the analysis variation and gives an indication of the precision of the test method; while the mean of replicates indicates the accuracy of the test method [25,26]. For determination of the percentage recovery of hydroxytyrosol in sample, it was added to a placebo sample at a specified concentration and analyzed using HPLC-DAD. Results showed the current method to achieve good recovery rates (from $98.8 \%$ to $100.1 \%$ ) for hydroxytyrosol at the three concentration levels studied (3, 25 and $100 \mathrm{ppm}$ ), and with an RSD under $0.5 \%$ (Table 1).

The precision of the analytical method was determined as inter and intra-day variability expressed as relative standard deviation (\%RSD). The precision of the current method was evaluated by calculating the $\mathrm{RSD} \%$ of the peak areas of six replicate injections of three standard solutions (3, 25 and $100 \mathrm{ppm}$ ) (Table 2).

LOD and LOQ: LOD was determined by preparing a solution that produced a response of about 3 and 10 times the baseline noise. The solution was injected three times, and the $\mathrm{S} / \mathrm{N}$ ratio recorded for each injection. Solution concentration is considered LOD if $\mathrm{S} / \mathrm{N}$ ratio is between 3-10. LOQ was determined in the same manner but with an S/N ratio of 10-20. The LOD and LOQ of hydroxytyrosol using this method were determined by preparing differently diluted solutions and injecting these into the liquid chromatograph, recording the $\mathrm{S} / \mathrm{N}$ ratio for hydroxytyrosol peak at each concentration. Results showed LOD and LOQ of hydroxytyrosol to be $2.49 \mathrm{ppm}$ and $3.97 \mathrm{ppm}$, respectively (Table 2).

Selectivity: The selectivity of the current method was demonstrated by a good separation of hydroxytyrosol from other interfering peaks (EVOO compounds and co-formulant agents) present in sample with good resolution. Figure 3 shows a representative chromatogram of the reference compound (3A) and sample analyzed (3B).

\section{Results and Discussion}

Preliminary studies were conducted to find the best analytical conditions to separate hydroxytyrosol using C8 and C18 columns and different compositions of the mobile phase. Parameters such as peak tailing minimized, good symmetry and good resolution between hydroxytyrosol and adjacent peaks were taken into account for the selection of analytical conditions. On this basis was chosen an Ascentis C18 column $(150 \times 4.6 \mathrm{~mm}, 5 \mu \mathrm{m})$ as stationary phase and a mixture consisting of acetic acid $0.2 \%$ and methanol (pH 3.30) as the mobile phase using a gradient elution program (see section 2.4). The best separation was obtained at a flow rate of $1.5 \mathrm{ml} / \mathrm{min}$ at a wavelength of $280 \mathrm{~nm}$.

The presence of acetic acid in the mobile phase achieves a very sharp peak for hydroxytyrosol, while the mobile phase without acid gives very wide peaks (low theoretical plates) and poor resolution.

Different organic solvents were also tested in order to keep analysis time to a minimum. Solvents tested included methanol, acetonitrile and ethanol with acetic acid solution. The best separation along with a short stroke chromatography was achieved using methanol as organic solvent, with acetic solution (run time 15 minutes). Table 1 shows

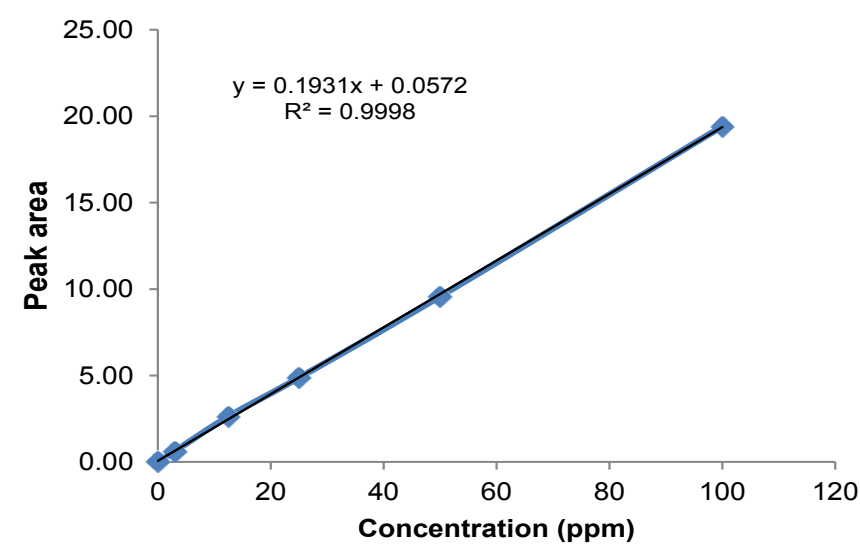

Figure 2: Calibration curve for hydroxytyrosol determination by the proposed method (area vs. concentration in ppm). 

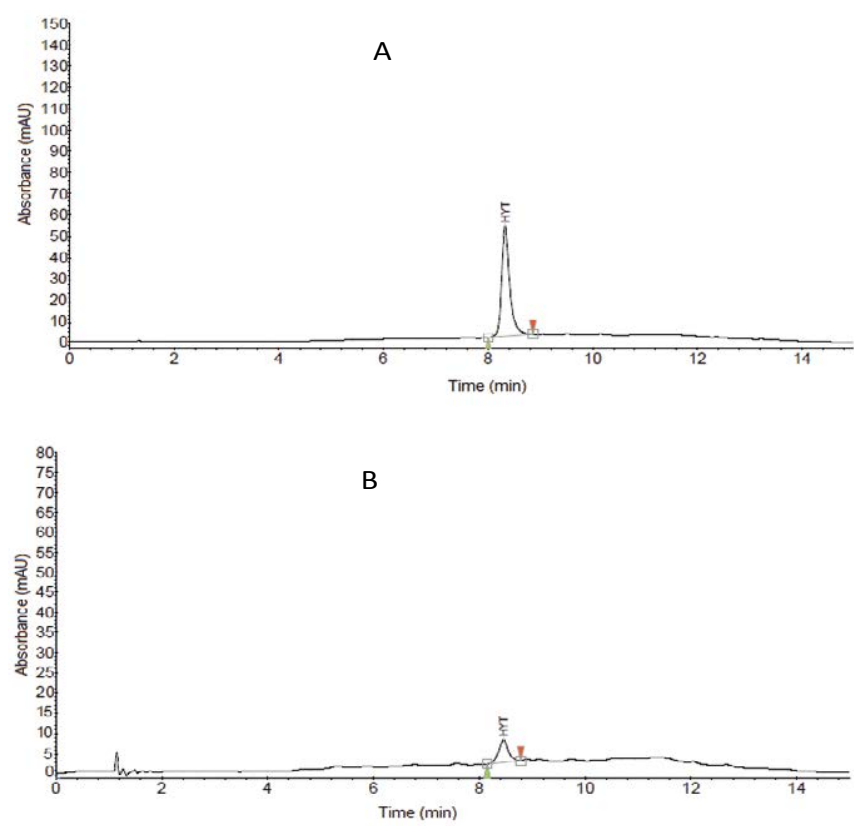

Figure 3: Representative chromatogram of reference compound $(A)$ and of semi-solid pharmaceutical formulation containing hydroxytyrosol (B) analyzed by the proposed method.

\begin{tabular}{|c|c|c|c|c|c|c|}
\hline \multirow{2}{*}{$\begin{array}{c}\text { Organic solvents } \\
\text { used in the mobile } \\
\text { phase* }\end{array}$} & \multicolumn{3}{|c|}{$\begin{array}{l}\text { C8 column Prodigy } \\
(150 \times 4.6 \mathrm{~mm}, 5 \mu \mathrm{m})\end{array}$} & \multicolumn{3}{|c|}{$\begin{array}{l}\text { C18 column Ascentis } \\
(150 \times 4.6 \mathrm{~mm}, 5 \mu \mathrm{m})\end{array}$} \\
\hline & $R s$ & $R t$ & Peak Shape & Rs & $R t$ & Peak Shape \\
\hline Methanol & 1.73 & 8.05 & Good & 2.30 & 8.45 & Good \\
\hline Acetonitrile & 1.45 & 7.54 & Poor & 1.54 & 7.78 & Poor \\
\hline Ethanol & 1.60 & 7.65 & Poor & 1.81 & 7.82 & Poor \\
\hline
\end{tabular}

*The organic solvent only was replaced in the gradient elution program reported in section 2.4

Table 1: Chromatography data of different solvents and stationary phases used for development and optimization of the analytical method. Rt: Retention time; Rs: Resolution.

the chromatographic parameters taken into account to develop and optimize the analytical method.

Hydroxytyrosol extraction from lipophilic samples was carried out using different concentrations of acid solution but the best results were obtained using acetic acid $0.2 \%$. The effect of extraction solvent $\mathrm{pH}$ and extraction volume were also studied. A sample cleaning step is needed before HPLC analysis and we tested various stationary phases, such as C18 Sep-pak and others (recovery $\leq 88.70 \%$ ). However, the best purification, in terms of recovery, was obtained using a Strata-X cartridge (see Table 2). In order to evaluate the extraction yield, a standard solution (containing $10 \mu \mathrm{g} \mathrm{mL} \mathrm{m}^{-1}$ of analyte) was analyzed directly and after performing the SPE extraction procedure under the conditions selected (see Section 2.3). The results were compared and showed that extraction yields of $100.5 \pm 3 \%$ for hydroxytyrosol (date not shown).

The quality of the analytical parameters of the chosen method was evaluated using standard solutions of hydroxytyrosol. At the range recorded (3.0 to $\left.100 \mu \mathrm{g} \mathrm{mL} \mathrm{mL}^{-1}\right)$ the method showed a high degree of linearity $\left(\mathrm{R}^{2}>0.9998\right)$.

The respective LOD and LOQ of this method were 2.49 and 3.97 ppm. These low limits of detection and quantification along with the broad linear range observed allow the target analyte to be determined in a variety of semi-solid pharmaceutical formulations containing different amounts of hydroxytyrosol.

The accuracy of the analytical method proposed, understood as measures of the agreement between the value, which is accepted either as a conventional true value or an accepted reference value, and the value found (i.e., accuracy is a measure of the exactness of an analytical method), showed a recovery rate $>$ of $98.8 \%$ and a RSD $\%$ value $>$ to $0.5 \%$ [25-27].

Furthermore, the method showed a good degree of precision (see Table 3). All experiments were performed in triplicate and repeated at least three times.

Recovery rates were evaluated by determining the analyte concentrations of both spiked and non-spiked samples applying the proposed method. The recovery rates obtained (Table 2 ) ranged from 98.6 to $100.2 \%$, thus showing that there was no matrix effect.

\section{Conclusions}

Hydroxytyrosol is a very interesting compound used as an ingredient in several pharmaceutical formulations due to its beneficial properties on skin. The proposed method provides a simple and effective means to determine hydroxytyrosol in a diverse range of samples, enabling the direct analysis of hydrophilic samples after dilution and filtration or after performing a liquid-liquid extraction for lipophilic samples. Thus, the proposed method can be considered safe for both the operator and the environment according to the principles of the 'Green Analytical Chemistry'. The proposed method is also useful in quality control of the whole industrial process to obtain the final pharmaceutical formulation. In the present study, a new formulation of topical cream containing hydroxytyrosol conveyed in EVOO was studied and this represents the most innovative aspect of our study, because for the first time a method has been developed and validated for the determination of hydroxytyrosol in a semi-solid formulation containing contextually extra virgin olive oil. Although EVOO contains several compounds structurally related to hydroxytyrosol; the method was found be selective in that hydroxytyrosol is well separated from other polyphenols naturally present in the EVOO with good resolution.

However, it is expected that the proposed method could be valid for the analysis of other creams containing the same active compound. It is worth mentioning that a very important step in the manufacture of a new cosmetic formulation is the study of its stability when stored under normal conditions. The antioxidant activity of hydroxytyrosol, like other polyphenols present in EVOO, contributes significantly

\begin{tabular}{|c|c|c|c|c|c|c|}
\hline $\begin{array}{c}\text { Concentration } \\
\text { (ppm) }\end{array}$ & \multicolumn{3}{|c|}{ Recovery (\%) } & Mean & SD & RSD (\%) \\
\hline 3 & 98.6 & 99.3 & 98.5 & 98.8 & 0.44 & 0.44 \\
\hline 25 & 100.1 & 99.3 & 100.2 & 99.9 & 0.49 & 0.49 \\
\hline 100 & 100.2 & 100.3 & 99.8 & 100.1 & 0.26 & 0.26 \\
\hline
\end{tabular}

RSD: Relative standard deviation; SD: Standard deviation.

Table 2: Percentage recovery of hydroxytyrosol at three different concentrations (2, 25 and $100 \mathrm{ppm})$.

\begin{tabular}{|c|c|c|c|c|}
\hline \multirow{2}{*}{ Compound } & \multicolumn{4}{|c|}{ Parameters } \\
\cline { 2 - 5 } & $\begin{array}{c}\text { RSD(\%), } \boldsymbol{n = 6} \\
\text { inter-day }\end{array}$ & $\begin{array}{c}\text { RSD (\%), } \boldsymbol{n = 6} \\
\text { intra-day }\end{array}$ & LOD (ppm) & LOQ (ppm) \\
\hline Hydroxytyrosol & 1.44 & 1.68 & 2.49 & 3.97 \\
\hline
\end{tabular}

RSD: Relative standard deviation; LOD: Limit of detection; LOQ: Limit of quantification

Table 3: Precision, LOD and LOQ of the proposed method. 
to the stability of this kind of semi-solid formulation at different temperatures and conditions of exposure to light. Therefore, the good analytical performance of the proposed method allows it also to be used for carrying out these studies.

\section{Acknowledgements}

The Authors are grateful to P\&P Farma S.r.I. (Torino, Italy) for providing semisolid pharmaceutical preparation samples containing a hydroxytyrosol extract conveyed in EVOO.

\section{References}

1. Tapsell LC (2014) Foods and food components in the Mediterranean diet: supporting overall effects. BMC Med 12: 100.

2. Martinez-Gonzalez MA, Salas-Salvado J, Estruch R, Corella D, Fito M, et al. (2015) Benefits of the Mediterranean Diet: Insights From the PREDIMED Study. Prog Cardiovasc Dis 58: 50-60.

3. Bendini A, Cerretani L, Carrasco-Pancorbo A, Gómez-Caravaca AM, SeguraCarretero A, et al. (2007) Phenolic Molecules in Virgin Olive Oils: A Survey of Their Sensory Properties, Health Effects, Antioxidant Activity and Analytical Methods. An Overview of the Last Decade Molecules 12: 1679-1719.

4. Hernáez Á, Fernández-Castillejo $S$, Farràs $M$, Catalán Ú, Subirana I, et al. (2014) Olive oil polyphenols enhance high-density lipoprotein function in humans: a randomized controlled trial. Arterioscler Thromb Vasc Biol 34: 21152119.

5. Medina-Remón A, Tresserra-Rimbau A, Pons A, Tur JA, Martorell M, et al. (2015) Effects of total dietary polyphenols on plasma nitric oxide and blood pressure in a high cardiovascular risk cohort. The PREDIMED randomized trial. Nutr Metab Cardiovasc Dis 25: 60-67.

6. Farzaei MH, Rahimi R, Abdollahi M (2015) The role of dietary polyphenols in the management of inflammatory bowel disease. Curr Pharm Biotechnol 16: $196-210$.

7. European Food Safety Authority (EFSA) (2001).

8. Martínez-Lapiscina EH, Clavero $P$, Toledo E, San Julián B, Sanchez-Tainta A, et al. (2013) Virgin olive oil supplementation and long-term cognition: the PREDIMED-NAVARRA randomized, trial. J Nutr Health Aging 17: 544-552.

9. Rosignoli P, Fuccelli R, Fabiani R, Servili M, Morozzi G (2013) Effect of olive oi phenols on the production of inflammatory mediators in freshly isolated human monocytes. J Nutr Biochem 24: 1513-1519.

10. Garcia-Martinez O, Rivas A, Ramos-Torrecillas J, De Luna-Bertos E, Ruiz C (2014) The effect of olive oil on osteoporosis prevention. Int J Food Sci Nutr 65: 834-840.

11. Coccia A, Bastianelli D, Mosca L, Monticolo R, Panuccio I, et al. (2014) Extra virgin olive oil phenols suppress migration and invasion of T24 human bladder cancer cells through modulation of matrix metalloproteinase-2. Nutr Cancer 66: 946-954.
12. Haloui E, Marzouk B, Marzouk Z, Bouraoui A, Fenina N (2011) Hydroxytyrosol and oleuropein from olive leaves: potent anti-inflammatory and analgesic activities. J Food Agric Environ 9: 128-133.

13. Hu T, He XW, Jiang JG, Xu XL (2014) Hydroxytyrosol and its potential therapeutic effects. J Agric Food Chem 62: 1449-1455.

14. Manna C, Galletti P, Maisto G, Cucciolla V, D'Angelo S, et al. (2000) Transport mechanism and metabolism of olive oil hydroxytyrosol in Caco-2 cells. FEBS Lett 470: 341-344.

15. D'Angelo S, Ingrosso D, Migliardi V, Sorrentino A, Donnarumma G, et al. (2005) Hydroxytyrosol, a natural antioxidant from olive oil, prevents protein damage induced by long-wave ultraviolet radiation in melanoma cells. Free Radic Biol Med 38: 908-919.

16. Salucci S, Burattini S, Curzi D, Buontempo F, Martelli AM, et al. (2014) Antioxidants in the prevention of UVB-induced keratynocyte apoptosis. J Photochem Photobiol B 141: 1-9.

17. Salucci S, Burattini S, Battistelli M, Buontempo F, Canonico B, et al. (2015) Tyrosol prevents apoptosis in irradiated keratinocytes. J Dermatol Sci.

18. Tomohiro C (2004) Whitening effect of hydroxytyrosol containing Oleaceae plants. Fragr J 32: 41-48.

19. Khan BA, Akhtar N, Mahmood T, Qayum M, Zaman SU (2010) Formulation and pharmaceutical evaluation of a W/O emulsion of Hippophae ramnoides fruit extract. J Pharm Res 3: 1342-1344.

20. Visioli F, Galli C, Grande S, Colonnelli K, Patelli C, et al. (2003) Hydroxytyroso excretion differs between rats and humans and depends on the vehicle of administration. J Nutr 133: 2612-2615.

21. Miralles P, Chisvert A, Salvador A (2015) Determination of hydroxytyrosol and tyrosol by liquid chromatography for the quality control of cosmetic products based on olive extracts. J Pharm Biomed Anal 102: 157-161.

22. International Conference on Harmonization of Technical Requirements for the Registration of Pharmaceuticals for Human Use (ICH) (1996) Validation of Analytical Procedures: Methodology, ICH-Q2B, Geneva.

23. United States Pharmacopeial Convention (2009) USP NF. U.S Pharmacopeia: Rockville.

24. International Conference on Harmonization (ICH) (2005) Validation of Analytical Procedures dPA/PH/OMCL (05) $47 \mathrm{DEF}$, elaborated by OMCL Network/EDQM of the Council of Europe.

25. Green JM (1996) Peer reviewed: a practical guide to analytical method validation. Anal Chem 68: 305A-309A.

26. Winslow PA, Meyer RF (1997) Defining a master plan for the validation of analytical methods. J Valid Technol 14: 361-367.

27. Wegscheider (1996) Validation of analytical methods. In: Guenzler H, editor Accreditation and quality assurance in analytical chemistry. Berlin: Springer Verlag. 